Rev. Mat. Iberoamericana 21 (2005), no. 2, 459-481

\title{
Some Remarks on the Weak Maximum Principle
}

Marco Rigoli, Maura Salvatori and Marco Vignati

\begin{abstract}
We obtain a maximum principle at infinity for solutions of a class of nonlinear singular elliptic differential inequalities on Riemannian manifolds under the sole geometrical assumptions of volume growth conditions. In the case of the Laplace-Beltrami operator we relate our results to stochastic completeness and parabolicity of the manifold.
\end{abstract}

Dedicated to the memory of Franca Burrone Rigoli.

\section{Introduction}

In what follows we let $(M,\langle\cdot, \cdot\rangle)$ denote a non-compact, connected Riemannian manifold, and $r(x)=\operatorname{dist}_{(M,\langle\cdot, \cdot)}(x, o)$ for some fixed reference point $o \in M$, so that $B_{R}$ is the geodesic ball of radius $R$ centered at $o$. A famous result of $\mathrm{H}$. Omori and $\mathrm{S}$. T. Yau asserts that for each $u \in \mathcal{C}^{2}(M)$ with $u^{*}=\sup _{M} u<+\infty$, there exists a sequence $\left\{x_{k}\right\} \subset M$ with the following properties: for each $k \in \mathbb{N}$,

$$
\text { i) } \left.u\left(x_{k}\right) \rightarrow u^{*} \quad \text { ii) } \Delta u\left(x_{k}\right)<\frac{1}{k} \quad \text { iii }\right)|\nabla u|\left(x_{k}\right)<\frac{1}{k}
$$

provided the metric is complete and some curvature assumptions are satisfied [2].

This result is known in the literature as the Omori-Yau Maximum Principle, and it has been used in the solution of a variety of geometrical problems since its appearance in the beautiful proof of the Schwarz's lemma for holomorphic maps between Kähler manifolds due to Yau himself [18].

2000 Mathematics Subject Classification: 58J05, 53C21.

Keywords: Maximum principles, volume growth. 
In some situation this principle is not applied in its full strength, in the sense that often the information (1.1) iii) does not need to be used. This is for instance the case when, according to an old question of Calabi, we study the existence of complete minimal surfaces in $\mathbb{R}^{3}$ with bounded image. Thus, the following definition seems to be appropriate:

Definition 1 Let $(M,\langle\cdot, \cdot\rangle)$ be a (not necessarily complete) Riemannian manifold. We say that $(M,\langle\cdot, \cdot\rangle)$ satisfies the weak maximum principle if for each $u \in \mathcal{C}^{2}(M), u^{*}<+\infty$, there exists a sequence $\left\{x_{k}\right\} \subset M$ with the properties (1.1) i); ii) above.

This seemingly simple minded definition is in fact surprisingly deep; indeed, it unexpectedly relates to stochastic completeness. As a matter of fact, in a recent paper, [9], the authors proved the equivalence of the following statements:

$$
(M,\langle\cdot, \cdot\rangle) \text { is stochastically complete; }
$$

$$
\forall \lambda>0 \text {, the differential equation } \Delta u=\lambda u \text { has no non- }
$$
negative, non-identically null bounded solutions;

$$
\begin{aligned}
\forall u & \in \mathcal{C}^{2}(M), u \text { non-constant, } u^{*}<+\infty \text { and } \forall \gamma<u^{*}, \\
& \inf _{\Omega_{\gamma}} \Delta u \leq 0, \text { with } \Omega_{\gamma}=\{x \in M: u(x)>\gamma\}
\end{aligned}
$$

$$
(M,\langle\cdot, \cdot\rangle) \text { satisfies the weak maximum principle. }
$$

We stress that the metric has not been assumed to be complete. Indeed, neither the weak maximum principle nor any of the above equivalent formulations require it.

The equivalence of (1.2) and (1.3) is well known, and due to Grigor'yan, see for instance [3].

We observe that, for $\lambda>0$, any positive constant is a super-solution of $\Delta u=\lambda u$ thus, using the Monotone Iteration Scheme on an appropriate exhaustion of $M,(1.3)$ can be equivalently substituted by

$$
\begin{aligned}
& \forall \lambda>0, \text { the differential inequality } \Delta u \geq \lambda u \text { has no } \\
& \text { non-negative, non-identically null bounded solutions. }
\end{aligned}
$$

This can also be seen by observing that the statement

$$
\begin{aligned}
& \forall f \in \mathcal{C}^{0}(\mathbb{R}) \text { and } \forall u \in \mathcal{C}^{2}(M) \text { with } u^{*}<+\infty \text { satisfying } \\
& \Delta u \geq f(u) \text { we have } f\left(u^{*}\right) \leq 0 .
\end{aligned}
$$

is implied by (1.5), and it implies (1.3), so that it is in fact equivalent to the above. 
To analyse $\left(1.3^{\prime}\right)$ a step further, we let $u$ be a subharmonic function on $M$ with $u^{*}<+\infty$. Without loss of generality we can suppose that $u^{*}>0$ and therefore, since $\sup \{0, u\}$ is still a subharmonic function, we can assume that $u$ is a non-negative, bounded subharmonic function. This shows that we can interpret $\left(1.3^{\prime}\right)$ with $\lambda=0$ just as the parabolicity of the manifold $(M,\langle\cdot, \cdot\rangle)$. To be completely precise, the following statements are equivalent:

$$
(M,\langle\cdot, \cdot\rangle) \text { is parabolic; }
$$

the differential inequality $\Delta u \geq 0$ has no non-constant, non-negative bounded solutions;

$\forall u \in \mathcal{C}^{2}(M), u$ non-constant, $u^{*}<+\infty$ and $\forall \gamma<u^{*}$, $\inf _{\Omega_{\gamma}} \Delta u \leq 0$, with $\Omega_{\gamma}=\{x \in M: u(x)>\gamma\}$;

$\forall u \in \mathcal{C}^{2}(M), u$ non-constant, $u^{*}<+\infty$, there exists a sequence $\left\{x_{k}\right\} \subset M$ such that, $\forall k \in \mathbb{N}$,

$$
u\left(x_{k}\right)>u^{*}-\frac{1}{k} ; \quad \Delta u\left(x_{k}\right)<0 .
$$

The only implication that needs some explanation is $(1.7) \Longrightarrow(1.9)$. (The equivalence of (1.7) with (1.8) having been pointed out above.)

Towards this aim let $u$ be as in (1.9) and assume that, for some $\gamma<u^{*}$, $\Delta u \geq 0$ on $\Omega_{\gamma}$. For $0<\varepsilon<u^{*}-\gamma$ we define

$$
v= \begin{cases}\gamma+\frac{\varepsilon}{2} & \text { on } M \backslash \overline{\Omega_{\gamma+\varepsilon / 2}} \\ u & \text { on } \overline{\Omega_{\gamma+\varepsilon}}\end{cases}
$$

Then $v=\sup \{u, \gamma+\varepsilon / 2\}$ on $\Omega_{\gamma} \backslash \overline{\Omega_{\gamma+\varepsilon}}$ and therefore $v$ is subharmonic on $M$. Because of (1.7) it follows that $v \equiv \gamma+\varepsilon / 2$ contradicting $u^{*}>\gamma+\varepsilon$.

Thus, $(M,\langle\cdot, \cdot\rangle)$ is parabolic if and only if it satisfies the somewhat stronger version of the weak maximum principle given in (1.10).

The above discussion shows the relevance of the study of appropriate formulations of the weak maximum principle. Motivated by the aim of investigating differential inequalities of the type (see Corollary 1.3 below)

$$
\Delta u \geq b(x) f(u)
$$

we look for a weak maximum principle in the following form:

$$
\begin{aligned}
& \text { given } \Lambda, \sigma \geq 0, \mu \in \mathbb{R} \text {, for each } u \in \mathcal{C}^{2}(M) \text {, u non- } \\
& \text { constant with } \hat{u}=\limsup _{r(x) \rightarrow+\infty} \frac{u(x)}{r(x)^{\sigma}}<+\infty \text {, if } \\
& \Omega_{\gamma}=\{x \in M: u(x)>\gamma\} \neq \emptyset \text { then: } \\
& \qquad \inf _{\Omega_{\gamma}}(1+r(x))^{\mu} \Delta u(x) \leq \Lambda \max \{\hat{u}, 0\} .
\end{aligned}
$$


We note that (1.11) can indeed be considered as some form of the weak maximum principle, in the sense that it reduces exactly to it in case $\mu=0$, $\sigma=0$ and $\Lambda=0$. However, we are now in general allowed to consider situations where $u^{*}=+\infty$.

Of course, it is essential to depict geometrical conditions to assure the validity of $(1.11)$ on $(M,\langle\cdot, \cdot\rangle)$ for appropriate values of $\Lambda, \sigma, \mu$. We recall that stochastic completeness and parabolicity, in case the metric is complete, are guaranteed, respectively, by the following volume growth assumptions:

$$
\frac{r}{\log \operatorname{vol}\left(B_{r}\right)} \notin L^{1}(+\infty) \quad ; \quad \frac{1}{\operatorname{vol}\left(\partial B_{r}\right)} \notin L^{1}(+\infty) .
$$

The first is, at present, the best general condition in this direction, and it has been proved by Grigor'yan (see for instance [3]). The second has been known for some time, and rediscovered by many researchers.

In the next result we shall require a more demanding type of volume growth assumption that, when $\mu=0$, is in some sense intermediate between the two above and which, however, is directly related to the value of $\Lambda$ in (1.11). We prove

Theorem 1.1 Let $(M,\langle\cdot, \cdot\rangle)$ be a complete manifold and let $\sigma, \mu \in \mathbb{R}$ satisfy

$$
\text { i) } \sigma \geq 0 ; \text { ii) } \sigma+\mu<2
$$

Assume that

$$
\liminf _{r \rightarrow+\infty} \frac{\log \operatorname{vol}\left(B_{r}\right)}{r^{2-\sigma-\mu}}=d_{0}<+\infty .
$$

Then, given $u \in \mathcal{C}^{2}(M)$ such that

$$
\hat{u}=\limsup _{r(x) \rightarrow+\infty} \frac{u(x)}{r(x)^{\sigma}}<+\infty
$$

and $\gamma \in \mathbb{R}$ with

$$
\Omega_{\gamma}=\{x \in M: u(x)>\gamma\} \neq \emptyset
$$

we have

$$
\inf _{\Omega_{\gamma}}[1+r(x)]^{\mu} \Delta u(x) \leq d_{0} \max \{\hat{u}, 0\} C(\sigma, \mu)
$$

with

$$
C(\sigma, \mu)= \begin{cases}0 & \text { if } \sigma=0 \\ (2-\sigma-\mu)^{2} & \text { if } \sigma>0,2(1-\sigma)>\mu \\ \sigma(2-\sigma-\mu) & \text { if } \sigma>0,2(1-\sigma) \leq \mu\end{cases}
$$


Condition (1.13) describes, broadly speaking, a volume growth of at most exponential type. For a growth of at most polynomial type we substitute (1.13) with

$$
\liminf _{r \rightarrow+\infty} \frac{\log \operatorname{vol}\left(B_{r}\right)}{\log r}=d_{0}<+\infty .
$$

In this case, with the notation of Theorem 1.1, we observe that, if $\sigma+\mu<2$, (1.17) implies the validity of (1.13) with $d_{0}=0$, so that

$$
\inf _{\Omega_{\gamma}}[1+r(x)]^{\mu} \Delta u(x) \leq 0 .
$$

The interesting cases left to analyse are therefore

$$
\text { i) } \sigma \geq 0 ; \text { ii) } \sigma+\mu=2
$$

Theorem 1.2 Let $(M,\langle\cdot, \cdot\rangle)$ be a complete manifold and assume (1.17) and (1.18). Then, given $u \in \mathcal{C}^{2}(M)$ satisfying (1.14) and $\gamma \in \mathbb{R}$ such that $\Omega_{\gamma} \neq \emptyset$ we have

$$
\inf _{\Omega_{\gamma}}[1+r(x)]^{\mu} \Delta u(x) \leq \sigma \max \{\hat{u}, 0\} C\left(\sigma, d_{0}\right)
$$

with

$$
C\left(\sigma, d_{0}\right)= \begin{cases}0 & \text { if } \sigma+d_{0}<2 \\ \sigma+d_{0}-2 & \text { if } \sigma+d_{0} \geq 2\end{cases}
$$

We shall consider sharpness of the constants $C(\sigma, \mu), C\left(\sigma, d_{0}\right)$ and of the volume growth assumptions in Section 3 below.

Corollary 1.3 Let $(M,\langle\cdot, \cdot\rangle)$ be a complete manifold and let $b \in \mathcal{C}^{0}(M)$ be such that

$$
\text { i) } b(x)>0 \quad \text { on } M \quad ; \quad \text { ii }) \quad b(x) \geq \frac{B}{r(x)^{\mu}} \quad \text { on } M \backslash B_{R_{0}}
$$

for some $R_{0}, B>0$ and $\mu \in \mathbb{R}$. Let $f \in \mathcal{C}^{0}(\mathbb{R})$ with

$$
\liminf _{t \rightarrow+\infty} f(t)>0 \text {. }
$$

Let $u \in \mathcal{C}^{2}(M)$ be a solution of

$$
\Delta u \geq b(x) f(u) \quad \text { on } M
$$

such that, for some $\sigma \geq 0$

$$
u(x)=o\left(r(x)^{\sigma}\right) \quad \text { as } r(x) \rightarrow+\infty .
$$

Assume that

$$
\sigma+\mu<2 \quad \text { and } \quad \liminf _{r \rightarrow+\infty} \frac{\log \operatorname{vol}\left(B_{r}\right)}{r^{2-\sigma-\mu}}<+\infty .
$$

Then $u^{*}<+\infty$ and $f\left(u^{*}\right) \leq 0$. 
It would be interesting to determine conditions on $f$ and the manifold $M$ to guarantee the validity of (1.21). This has been done, for instance, in Theorem 2.6 of [13] where, improving an original result of Cheng and Yau, it is shown that $u^{*}<+\infty$ under curvature assumptions, $b(x) \equiv 1$ and, basically, $f$ is eventually positive and superlinear. However, under sole volume growth conditions as geometrical requirements, the task is really hard. In the recent paper [10] the authors succeeded in proving an $L^{\infty}$ bound for non-negative solutions $u$ of

$$
\Delta u \geq b(x) u^{\gamma}-a(x) u, \quad \gamma>1
$$

under volume growth assumptions and conditions on the behaviour of the coefficients $a(x)$ and $b(x)$. The technique devised in that paper can also be used to prove a version of Theorem 1.1 but only, in the present notations, for $\sigma=0$ and $0 \leq \mu<2$. We also would like to recall that the first result in this direction is due to L. Karp, [8].

A second aim of this paper is to extend Theorems 1.1 and 1.2 and Corollary 1.3 to a large class of differential operators, defined in Section 2 below, such as, for instance, the $p$-Laplacian and the mean curvature operator. This extension has strong geometrical motivations. To quote a few, we refer to the recent works of Holopainen [6], [7]. Our class of operators essentially coincides with those considered by Serrin and collaborators, see for instance from the seminal paper [16] to the recent [11], [12], and this class has been intensively studied in this last decade. Results in this direction, especially on a generalization of the notion of parabolicity, have been considered in [6] and [14]. Our techniques can also be applied to Markov energy measure spaces and corresponding differential operators, with some assumptions. For this topic we refer to [17], [1], and [4], where beautiful applications to the geometry of minimal surfaces are presented. We shall pursue this direction in a forthcoming paper.

\section{Proofs and generalizations of the results}

Geometrical problems, for instance the study of assigned mean curvature graphs on a complete manifold, or non-linear potential theory, point out the interesting goal of extending the analysis to a larger class of differential operators in divergence form, other than the Laplacian. Towards this aim we let $\varphi \in \mathcal{C}^{1}((0,+\infty)) \cap \mathcal{C}^{0}([0,+\infty))$ satisfy the following structural conditions:

$$
\text { i) } \varphi(0)=0 ; \quad \text { ii) } \varphi(t)>0 \text { if } t>0 ; \quad \text { iii) } \varphi(t) \leq A t^{\delta} \text { if } t \geq 0
$$

for some positive constants $A$ and $\delta$. 
We shall focus our attention on the differential operator defined, for $u \in \mathcal{C}^{1}(M)$, by

$$
\operatorname{div}(T u) \equiv \operatorname{div}\left(|\nabla u|^{-1} \varphi(|\nabla u|) \nabla u\right)
$$

and which we shall refer to as the $\varphi-$ Laplacian. Of course, if the vector field in brackets is not $\mathcal{C}^{1}$ the divergence in (2.2) must be considered in distributional sense. Note that the vector field in consideration may fail to be $\mathcal{C}^{1}$ at the points where $\nabla u=0$ even if $u$ is assumed to be $\mathcal{C}^{2}$.

As important natural examples we mention:

i) the Laplace-Beltrami operator $\Delta u$, corresponding to $\varphi(t)=t$;

ii) more generally, the $p$-Laplacian operator $\operatorname{div}\left(|\nabla u|^{p-2} \nabla u\right), p>1$, corresponding to $\varphi(t)=t^{p-1}$;

iii) the generalized mean curvature operator $\operatorname{div}\left(\frac{\nabla u}{\left(1+|\nabla u|^{2}\right)^{\alpha}}\right), \alpha>0$, corresponding to $\varphi(t)=\frac{t}{\left(1+t^{2}\right)^{\alpha}}$.

We mention that the usual maximum principle and the Compact Support Property have been recently studied (under some further assumptions on $\varphi$ ) for instance by Pucci and Serrin, [11].

In the next statements we shall always assume that $\varphi \in \mathcal{C}^{1}((0,+\infty)) \cap$ $\mathcal{C}^{0}([0,+\infty))$ satisfies the structural conditions $(2.1)$.

Theorem 2.1 Let $(M,\langle\cdot, \cdot\rangle)$ be a complete manifold, and let $\sigma, \mu \in \mathbb{R}$ satisfy

$$
\sigma>0 ; \delta \sigma+\mu<1+\delta \text {. }
$$

Assume that

$$
\liminf _{r \rightarrow+\infty} \frac{\log \operatorname{vol}\left(B_{r}\right)}{r^{1+\delta-\delta \sigma-\mu}}=d_{0}<+\infty
$$

Then, given $u \in \mathcal{C}^{2}(M)$ for which $T u \equiv|\nabla u|^{-1} \varphi(|\nabla u|) \nabla u$ is at least of class $\mathcal{C}^{1}$, and such that

$$
\hat{u}=\limsup _{r(x) \rightarrow+\infty} \frac{u(x)}{r(x)^{\sigma}}<+\infty
$$

and given $\gamma \in \mathbb{R}$ with

$$
\Omega_{\gamma}=\{x \in M: u(x)>\gamma\} \neq \emptyset
$$


we have

$(2.5) \inf _{\Omega_{\gamma}}[1+r(x)]^{\mu} \operatorname{div}(T u)(x) \leq A\left(\frac{2}{1+\delta}\right)^{1+\delta} \delta^{\delta} d_{0}[\max (\hat{u} ; 0)]^{\delta} C(\sigma, \mu, \delta)$ with

$$
C(\sigma, \mu, \delta)=\left\{\begin{array}{cc}
(1+\delta-\delta \sigma-\mu) \sigma^{\delta} & \text { if }(1+\delta)(1-\sigma) \leq \mu \\
(1+\delta-\delta \sigma-\mu)^{1+\delta} & \text { if }(1+\delta)(1-\sigma)>\mu
\end{array}\right.
$$

Proof: It will be convenient to use the notation

$$
\beta \equiv(1+\delta)(1-\sigma)-\mu=(1+\delta-\delta \sigma-\mu)-\sigma
$$

in terms of this notation, recall that we are working with

$$
\beta \in \mathbb{R} ; \sigma>0 ; \beta+\sigma>0 .
$$

Moreover, we note that (2.5) is an estimate from above of the quantity

$$
K \equiv \inf _{\Omega_{\gamma}}[1+r(x)]^{\mu} \operatorname{div}(T u)(x)
$$

and the proof is needed only in case $K>0$. Hence, we assume that

$$
[1+r(x)]^{\mu} \operatorname{div}(T u)(x) \geq K>0 \quad \text { on } \Omega_{\gamma} .
$$

Let $b>\max (\hat{u} ; 0)$; since $\sigma>0$, we may translate the function $u$ by a constant, so that $\hat{u}$ remains unchanged, and

$$
\frac{u(x)}{[1+r(x)]^{\sigma}}<b \text { on } M ; u\left(x_{0}\right)>0
$$

for some $x_{0} \in M$. Moreover, $\operatorname{div}(T u)$ is invariant under translation, the left hand side of (2.5) is increasing with $\gamma$, and the right hand side does not depend on $\gamma$; hence we may assume $\gamma \geq 0$.

Now, fix $\vartheta \in\left(\frac{1}{2}, 1\right)$; for any $R>0$ such that $B_{R} \cap \Omega_{\gamma} \neq \emptyset$ let $\psi \in \mathcal{C}^{\infty}(M)$ be a cut-off function such that

$$
\begin{array}{ll}
\text { i) } 0 \leq \psi \leq 1 ; & \text { ii) } \psi \equiv 1 \text { on } B_{\vartheta R} ; \\
\text { iii) } \psi \equiv 0 \text { on } M \backslash B_{R} ; & \text { iv) }|\nabla \psi| \leq \frac{c}{R(1-\vartheta)}
\end{array}
$$

for some absolute constant $c>0$.

Next, we choose $\lambda \in \mathcal{C}^{\infty}(\mathbb{R})$ with the following properties

$$
\begin{array}{ll}
\text { i) } 0 \leq \lambda \leq 1 ; & \text { ii) } \lambda \equiv 0 \text { on }(-\infty, \gamma] \\
\text { iii) } \lambda>0 \text { on }(\gamma,+\infty) ; & \text { iv) } \lambda^{\prime} \geq 0 \text { on }(\gamma,+\infty)
\end{array}
$$


We introduce the auxiliary function

$$
v=2 b(1+r)^{\sigma}-u
$$

and notice that it satisfies

$$
b(1+r)^{\sigma} \leq v \leq 2 b(1+r)^{\sigma} \text { on } \Omega_{\gamma} .
$$

With all these ingredients, we define the vector field

$$
W=\psi^{1+\delta} \lambda(u) F(v, r) T u
$$

where the function $F:[0,+\infty) \times[0,+\infty) \rightarrow(0,+\infty)$ is given by

$$
\begin{array}{lll}
\text { i) } & F(v, r)=F(v)=\exp \left[-q v^{1+(\beta / \sigma)}\right] & \text { if } \beta \leq 0 \\
\text { ii) } & F(v, r)=\exp \left[-q(1+r)^{\beta} v\right] & \text { if } \beta>0
\end{array}
$$

where $q>0$ is a constant (possibly different in the two cases) to be fixed later.

We notice that, in both cases, $\partial F / \partial v \equiv F_{v}<0$; moreover, the vector field $W$ vanishes on $\partial\left(B_{R} \cap \Omega_{\gamma}\right)$, so that, by the Divergence Theorem,

$$
\int_{B_{R} \cap \Omega_{\gamma}} \operatorname{div} W=0 \text {. }
$$

Computing the divergence of $W$ we obtain:

$$
\begin{aligned}
\operatorname{div} W & =(1+\delta) \psi^{\delta} \lambda(u) F(v, r)\langle\nabla \psi, T u\rangle+\psi^{1+\delta}\left\{\lambda^{\prime}(u) F(v, r)\langle\nabla u, T u\rangle\right. \\
& \left.+\lambda(u)\left[F_{v}\langle\nabla v, T u\rangle+F_{r}\langle\nabla r, T u\rangle\right]+\lambda(u) F(v, r) \operatorname{div}(T u)\right\} .
\end{aligned}
$$

The Cauchy-Schwarz inequality, properties (2.10), (2.11) and (2.14) lead to

$$
\begin{aligned}
\operatorname{div} W \geq & -(1+\delta) \psi^{\delta} \lambda(u) F(v, r) \varphi(|\nabla u|)|\nabla \psi|+ \\
& +\psi^{1+\delta} \lambda(u)\left|F_{v}\right|\left\{\frac{F}{\left|F_{v}\right|} K(1+r)^{-\mu}+|\nabla u| \varphi(|\nabla u|)+\right. \\
& \left.+\left[\frac{F_{r}}{\left|F_{v}\right|}-2 b \sigma(1+r)^{\sigma-1}\right]|\nabla u|^{-1} \varphi(|\nabla u|)\langle\nabla r, \nabla u\rangle\right\} .
\end{aligned}
$$

Using (2.14) and (2.12) we estimate in both cases the quantity within the square brackets

$$
\left[\frac{F_{r}}{\left|F_{v}\right|}-2 b \sigma(1+r)^{\sigma-1}\right] \geq\left\{\begin{array}{cl}
(-\sigma) 2 b(1+r)^{\sigma-1} & \text { if } \beta \leq 0 \\
-(\beta+\sigma) 2 b(1+r)^{\sigma-1} & \text { if } \beta>0
\end{array},\right.
$$


and the quantity

$$
\frac{F}{\left|F_{v}\right|} \geq\left\{\begin{array}{cl}
\frac{\sigma}{q(\beta+\sigma)} b^{-\beta / \sigma}(1+r)^{-\beta} & \text { if } \beta \leq 0 \\
\frac{(1+r)^{-\beta}}{q} & \text { if } \beta>0
\end{array}\right.
$$

and we note that from the structural condition (2.1)iii) we have the inequality

$$
|\nabla u| \varphi(|\nabla u|) \geq A^{-1 / \delta} \varphi(|\nabla u|)^{(\delta+1) / \delta} .
$$

Hence, from (2.16)-(2.19) we obtain

$$
\begin{aligned}
\operatorname{div} W \geq & -(1+\delta) \psi^{\delta} \lambda(u) F(v, r) \varphi(|\nabla u|)|\nabla \psi| \\
& +\psi^{1+\delta} \lambda(u)\left|F_{v}\right|\left\{A^{-\frac{1}{\delta}} \varphi(|\nabla u|)^{1+\frac{1}{\delta}}-2 b \sigma(1+r)^{\sigma-1} \varphi(|\nabla u|)\right. \\
& \left.+\frac{K \sigma}{q(\beta+\sigma)} b^{-\beta / \sigma}(1+r)^{-\beta-\mu}\right\}
\end{aligned}
$$

if $\beta \leq 0$, and

$$
\begin{gathered}
\operatorname{div} W \geq-(1+\delta) \psi^{\delta} \lambda(u) F(v, r) \varphi(|\nabla u|)|\nabla \psi| \\
+\psi^{1+\delta} \lambda(u)\left|F_{v}\right|\left\{A^{-\frac{1}{\delta}} \varphi(|\nabla u|)^{1+\frac{1}{\delta}}-2 b(\beta+\sigma)(1+r)^{\sigma-1} \varphi(|\nabla u|)\right. \\
\left.+\frac{K}{q}(1+r)^{-\beta-\mu}\right\}
\end{gathered}
$$

if $\beta>0$. Moreover, we can estimate from below the quantities within the braces in (2.20) and (2.21).

Claim: For $0<\tau<1$ and for any $x \in M$ :

i) the choices

$$
0<\Lambda \leq \frac{1-\tau}{2 A^{1 / \delta}} \quad ; \quad q=\frac{\tau^{\delta} K(1+\delta)^{1+\delta} \sigma b^{-\beta / \sigma}}{A \delta^{\delta}(2 b \sigma)^{1+\delta}(\beta+\sigma)}
$$

imply

$A^{-\frac{1}{\delta}} \varphi(|\nabla u|)^{1+\frac{1}{\delta}}-2 b \sigma(1+r)^{\sigma-1} \varphi(|\nabla u|)+\frac{K \sigma b^{-\beta / \sigma}}{q(\beta+\sigma)}(1+r)^{-\beta-\mu} \geq \Lambda \varphi(|\nabla u|)^{1+\frac{1}{\delta}}$

ii) the choices

$$
0<\Lambda \leq \frac{1-\tau}{2 A^{\frac{1}{\delta}}} \quad ; \quad q=\frac{\tau^{\delta} K(1+\delta)^{1+\delta}}{A \delta^{\delta}[2 b(\beta+\sigma)]^{1+\delta}}
$$

imply

$$
A^{-\frac{1}{\delta}} \varphi(|\nabla u|)^{1+\frac{1}{\delta}}-2 b(\beta+\sigma)(1+r)^{\sigma-1} \varphi(|\nabla u|)+\frac{K}{q}(1+r)^{-\beta-\mu} \geq \Lambda \varphi(|\nabla u|)^{1+\frac{1}{\delta}} .
$$


Proof of the Claim: It is an easy Calculus exercise to show that if $M, N, P$ are positive constants, and if

$$
0<\Lambda \leq M-\frac{\delta N^{1+\frac{1}{\delta}}}{(1+\delta)^{1+\frac{1}{\delta}} P^{\frac{1}{\delta}}}
$$

then

$$
M t^{1+\frac{1}{\delta}}-N t+P \geq \Lambda t^{1+\frac{1}{\delta}}
$$

for any $t \geq 0$.

Hence, the use of the Claim and the choices (2.22), (2.23) allow to obtain, from (2.20) and (2.21), the common estimate

$\operatorname{div} W \geq-(1+\delta) \psi^{\delta} \lambda(u) F(v, r) \varphi(|\nabla u|)|\nabla \psi|+\Lambda \psi^{1+\delta} \lambda(u)\left|F_{v}\right| \varphi(|\nabla u|)^{1+\frac{1}{\delta}}$ and from (2.15) it follows

$$
\begin{aligned}
\Lambda \int_{B_{R} \cap \Omega_{\gamma}} & \psi^{1+\delta} \lambda(u)\left|F_{v}\right| \varphi(|\nabla u|)^{1+\frac{1}{\delta}} \\
& \leq(1+\delta) \int_{B_{R} \cap \Omega_{\gamma}} \psi^{\delta} \lambda(u) F(v, r) \varphi(|\nabla u|)|\nabla \psi| .
\end{aligned}
$$

We now replace the correct expressions for $F_{v}$ obtained from (2.14), and make use of Hölder's inequality with conjugate exponents $1+\delta, 1+1 / \delta$ to obtain the chain of inequalities

$$
\begin{aligned}
& \int_{B_{R} \cap \Omega_{\gamma}} \psi^{1+\delta} \lambda(u) F(v, r)(1+r)^{\beta} \varphi(|\nabla u|)^{1+\frac{1}{\delta}} \\
& \leq c \int_{B_{R} \cap \Omega_{\gamma}} \psi^{\delta} \lambda(u) F(v, r) \varphi(|\nabla u|)|\nabla \psi| \\
& \leq c\left(\int_{B_{R} \cap \Omega_{\gamma}} \psi^{1+\delta} \lambda(u) F(v, r)(1+r)^{\beta} \varphi(|\nabla u|)^{1+\frac{1}{\delta}}\right)^{\delta /(1+\delta)} \times \\
& \times\left(\int_{B_{R} \cap \Omega_{\gamma}} \lambda(u) F(v, r)(1+r)^{-\beta \delta}|\nabla \psi|^{1+\delta}\right)^{1 /(1+\delta)}
\end{aligned}
$$

whence

$$
\begin{aligned}
\int_{B_{R} \cap \Omega_{\gamma}} & \psi^{1+\delta} \lambda(u) F(v, r)(1+r)^{\beta} \varphi(|\nabla u|)^{1+\frac{1}{\delta}} \\
& \leq c \int_{B_{R} \cap \Omega_{\gamma}} \lambda(u) F(v, r)(1+r)^{-\beta \delta}|\nabla \psi|^{1+\delta} .
\end{aligned}
$$


Having fixed $d>d_{0}$, according to (2.3) there exists a sequence $\left\{R_{k}\right\} \uparrow+\infty$ with $\vartheta R_{k+1}>R_{k}$ such that

$$
\log \operatorname{vol}\left(B_{R_{k}}\right) \leq d R_{k}^{\beta+\sigma} .
$$

Using (2.9), (2.10) and (2.25) with $R=R_{k}$ we have

$$
\begin{aligned}
& c \int_{B_{R_{1}} \cap \Omega_{\gamma}} \lambda(u) F(v, r) \varphi(|\nabla u|)^{1+\frac{1}{\delta}} \\
& \leq R_{k}^{-1-\delta} \int_{\left(B_{R_{k}} \backslash B_{\vartheta R_{k}}\right) \cap \Omega_{\gamma}} F(v, r)(1+r)^{-\beta \delta} .
\end{aligned}
$$

The condition $K>0$ implies that $u$ is not constant on the connected components of $B_{R_{1}} \cap \Omega_{\gamma}$, thus the left hand side of this inequality is strictly positive.

Hence, in case $\beta \leq 0$ from (2.14)i) and (2.26) one gets

$$
0<\liminf _{k \rightarrow+\infty}\left(1+R_{k}\right)^{-1-\delta-\beta \delta} \exp \left\{d R_{k}^{\beta+\sigma}-q b^{1+(\beta / \sigma)}\left(\vartheta R_{k}\right)^{\beta+\sigma}\right\}
$$

where $q$ is taken as in (2.22); this forces

$$
d>q b^{1+(\beta / \sigma)} \vartheta^{\beta+\sigma} .
$$

Letting $\vartheta, \tau \rightarrow 1^{-}, d \rightarrow d_{0}^{+}$we obtain

$$
d \geq \frac{(1+\delta)^{1+\delta} b \sigma K}{A \delta^{\delta}(2 b \sigma)^{1+\delta}(\beta+\sigma)}
$$

and when $b \downarrow \max (\hat{u} ; 0)$ this implies, recalling (2.6),

$$
K \leq A\left(\frac{2}{1+\delta}\right)^{1+\delta} \delta^{\delta} d_{0}[\max (\hat{u} ; 0)]^{\delta}(1+\delta-\delta \sigma-\mu) \sigma^{\delta}
$$

thus ending the proof for the case $\beta \leq 0$.

In case $\beta>0$ we use (2.14) ii) and (2.26) to obtain, from (2.27),

$$
0<\liminf _{k \rightarrow+\infty}\left(1+R_{k}\right)^{-1-\delta-\beta \delta} \exp \left\{d R_{k}^{\beta+\sigma}-q b\left(\vartheta R_{k}\right)^{\beta+\sigma}\right\}
$$

with $q$ as in (2.23). As above, we get

$$
K \leq A\left(\frac{2}{1+\delta}\right)^{1+\delta} \delta^{\delta} d_{0}[\max (\hat{u} ; 0)]^{\delta}(1+\delta-\delta \sigma-\mu)^{1+\delta}
$$

Our next result deals with the same kind of estimate, and covers the case when, in the assumptions of Theorem 2.1, $\sigma$ is null. Part of the proof resembles the previous one, and we shall avoid repetitions, when obvious. 
Theorem 2.2 Let $(M,\langle\cdot, \cdot\rangle)$ be a complete manifold, and $\mu<1+\delta$. Assume that

$$
\liminf _{r \rightarrow+\infty} \frac{\log \operatorname{vol}\left(B_{r}\right)}{r^{1+\delta-\mu}}=d_{0}<+\infty .
$$

Then, given $u \in \mathcal{C}^{2}(M)$ for which $T u \equiv|\nabla u|^{-1} \varphi(|\nabla u|) \nabla u$ is at least of class $\mathcal{C}^{1}$, and such that

$$
u^{*}=\sup _{M} u<+\infty
$$

and given $\gamma<u^{*}$ with $\Omega_{\gamma}=\{x \in M: u(x)>\gamma\}$ we have

$$
\inf _{\Omega_{\gamma}}[1+r(x)]^{\mu} \operatorname{div}(T u)(x) \leq 0 .
$$

Proof: Let

$$
\beta=1+\delta-\mu>0 .
$$

Since $\operatorname{div}(T u)$ is translation invariant, we may assume $u^{*}=0$. For any $\gamma<0$, the set $\Omega_{\gamma}=\{x \in M: u(x)>\gamma\}$ is non-empty, and if, as in (2.7) and (2.8)

$$
0<K \equiv \inf _{\Omega_{\gamma}}[1+r(x)]^{\mu} \operatorname{div}(T u)(x)
$$

the function $u$ is not constant on $\Omega_{\gamma}$. We shall prove that this leads to a contradiction.

Again, fix $\vartheta \in\left(\frac{1}{2}, 1\right)$, and choose $R>0$ so that $B_{R} \cap \Omega_{\gamma} \neq \emptyset$; let $\psi$ and $\lambda$ be cut-off functions as in (2.9) and (2.10) and define, for any $\eta>0$, the auxiliary function

$$
v(x)=\eta-u(x)
$$

that satisfies

$$
\eta \leq v(x) \leq \eta-\gamma \quad \text { on } \Omega_{\gamma}
$$

For

$$
F(v, r)=\exp \left[-q(1+r)^{\beta} v\right], \quad q>0
$$

as in (2.14) $i$ i) the same vector field used in (2.13)

$$
W=\psi^{1+\delta} \lambda(u) F(v, r) T u
$$

vanishes on $\partial\left(B_{R} \cap \Omega_{\gamma}\right)$, so that, by the Divergence Theorem,

$$
\int_{B_{R} \cap \Omega_{\gamma}} d i v W=0 .
$$


As in the previous proof, the computation of div $W$ leads to

$$
\begin{gathered}
\operatorname{div} W \geq-(1+\delta) \psi^{\delta} \lambda(u) F(v, r) \varphi(|\nabla u|)|\nabla \psi| \\
+\psi^{1+\delta} \lambda(u)\left|F_{v}\right|\left\{A^{-\frac{1}{\delta}} \varphi(|\nabla u|)^{1+\frac{1}{\delta}}-\beta(\eta-\gamma)(1+r)^{-1} \varphi(|\nabla u|)+\right. \\
\left.+\frac{K}{q}(1+r)^{-1-\delta}\right\}
\end{gathered}
$$

which is the analogue of (2.21).

Using part $i i$ ) of the Claim, the choices

$$
\text { i) } 0<\Lambda \leq \frac{1-\tau}{2 A^{1 / \delta}} \quad ; \quad \text { ii) } \quad q=\frac{\tau^{\delta} K(1+\delta)^{1+\delta}}{A \delta^{\delta}[\beta(\eta-\gamma)]^{1+\delta}}
$$

imply

$A^{-\frac{1}{\delta}} \varphi(|\nabla u|)^{1+\frac{1}{\delta}}-\beta(\eta-\gamma)(1+r)^{-1} \varphi(|\nabla u|)+\frac{K}{q}(1+r)^{-1-\delta} \geq \Lambda \varphi(|\nabla u|)^{1+\frac{1}{\delta}}$

whence

$$
\begin{aligned}
\Lambda \int_{B_{R} \cap \Omega_{\gamma}} & \psi^{1+\delta} \lambda(u)\left|F_{v}\right| \varphi(|\nabla u|)^{1+\frac{1}{\delta}} \\
& \leq(1+\delta) \int_{B_{R} \cap \Omega_{\gamma}} \psi^{\delta} \lambda(u) F(v, r) \varphi(|\nabla u|)|\nabla \psi|
\end{aligned}
$$

which is the same relation contained in (2.24).

Again, having fixed $d>d_{0}$ and having chosen the sequence $\left\{R_{k}\right\}$ as above, we obtain

$$
0<\liminf _{k \rightarrow+\infty}\left(1+R_{k}\right)^{-1-\delta-\beta \delta} \exp \left\{d R_{k}^{\beta}-q \eta\left(\vartheta R_{k}\right)^{\beta}\right\}
$$

where $q$ is given in (2.30), and this implies, letting $\vartheta \rightarrow 1^{-}$,

$$
d \geq \eta q=\frac{\tau^{\delta} K(1+\delta)^{1+\delta} \eta}{A \delta^{\delta}[(\eta-\gamma) \beta]^{1+\delta}}
$$

or, equivalently (letting $\tau \rightarrow 1^{-}$),

$$
K \leq \frac{A d \delta^{\delta} \beta^{1+\delta}(\eta-\gamma)^{1+\delta}}{(1+\delta)^{1+\delta} \eta}
$$

The quantity $K$ in non-decreasing with $\gamma$, while the right hand side of (2.31) is non-increasing, and so

$$
K \leq \lim _{\gamma \rightarrow 0^{-}} \frac{A d \delta^{\delta} \beta^{1+\delta}(\eta-\gamma)^{1+\delta}}{(1+\delta)^{1+\delta} \eta}=\frac{A d \delta^{\delta} \beta^{1+\delta} \eta^{\delta}}{(1+\delta)^{1+\delta}} .
$$

It now suffices to let $\eta \rightarrow 0^{+}$to contradict (2.29). 
Now, Theorem 1.1 of the Introduction is a special case of Theorems 2.1 and 2.2 since, in this case, $\varphi(t)=t$, and $A=\delta=1$.

Theorems 2.1 and 2.2 deal with the case when $(M,\langle\cdot, \cdot\rangle)$ has volume growth of at most exponential type of order $1+\delta-\delta \sigma-\mu>0$, meaning with this precisely condition (2.3). We are now concerned with the case when

$$
\liminf _{r \rightarrow+\infty} \frac{\log \operatorname{vol}\left(B_{r}\right)}{\log r}=d_{0}<+\infty
$$

that is, the manifold has at most polynomial growth (of order $d_{0}$ ).

Given $\mu \in \mathbb{R}$ and $\sigma \geq 0$, suppose $u \in \mathcal{C}^{2}(M)$ is such that

$$
T u \equiv|\nabla u|^{-1} \varphi(|\nabla u|) \nabla u
$$

is a vector field of at least of class $\mathcal{C}^{1}$ and

$$
\hat{u}=\limsup _{r(x) \rightarrow+\infty} \frac{u(x)}{r(x)^{\sigma}}<+\infty \text {. }
$$

If $\delta \sigma+\mu<1+\delta,(2.32)$ implies the validity of (2.3) with $d_{0}=0$. It therefore follows from (2.5) and (2.28) that

$$
\inf _{\Omega_{\gamma}}[1+r(x)]^{\mu} \operatorname{div}(T u)(x) \leq 0
$$

whenever $\Omega_{\gamma} \neq \emptyset$. The interesting cases left to analyse are therefore

$$
\sigma \geq 0 ; \delta \sigma+\mu=1+\delta .
$$

Theorem 2.3 Let $(M,\langle\cdot, \cdot\rangle)$ be a complete manifold, and let $\sigma, \mu \in \mathbb{R}$ satisfy

$$
\sigma \geq 0 ; \delta \sigma+\mu=1+\delta .
$$

Assume (2.32). Then, given $u \in \mathcal{C}^{2}(M)$ for which $T u \equiv|\nabla u|^{-1} \varphi(|\nabla u|) \nabla u$ is at least of class $\mathcal{C}^{1}$, and such that (2.33) holds, and given $\gamma \in \mathbb{R}$ with $\Omega_{\gamma} \neq \emptyset$, we have

$$
\inf _{\Omega_{\gamma}}[1+r(x)]^{\mu} \operatorname{div}(T u)(x) \leq A\left(\frac{2}{1+\delta}\right)^{1+\delta} \sigma^{\delta} \delta^{\delta}[\max (\hat{u} ; 0)]^{\delta} C\left(\sigma, \delta, d_{0}\right)
$$

with

$$
C\left(\sigma, \delta, d_{0}\right)= \begin{cases}\delta(\sigma-1)+d_{0}-1 & \text { if } \delta(\sigma-1)+d_{0}-1 \geq 0 \\ 0 & \text { if } \delta(\sigma-1)+d_{0}-1<0\end{cases}
$$


Proof: In case $\sigma>0$, we follow the reasoning of Theorem 2.1 with the same notations up to (2.12) included. Next, we choose

$$
F(v, r)=F(v)=v^{-q}
$$

with $q>0$ to be specified later. Again we have

$$
\begin{aligned}
{\left[\frac{F_{r}}{\left|F^{\prime}(v)\right|}-2 b \sigma(1+r)^{\sigma-1}\right]|\nabla u|^{-1} } & \varphi(|\nabla u|)\langle\nabla r, \nabla u\rangle \\
& \geq-2 b \sigma(1+r)^{\sigma-1} \varphi(|\nabla u|)
\end{aligned}
$$

while, using (2.12)

$$
\frac{F(v)}{\left|F^{\prime}(v)\right|}=\frac{v}{q} \geq \frac{b}{q}(1+r)^{\sigma}
$$

From (2.16) we thus deduce

$$
\begin{gathered}
\operatorname{div} W \geq-(1+\delta) \psi^{\delta} \lambda(u) F(v, r) \varphi(|\nabla u|)|\nabla \psi| \\
+\psi^{1+\delta} \lambda(u)\left|F^{\prime}(v)\right|\left\{A^{-1 / \delta} \varphi(|\nabla u|)^{1+\frac{1}{\delta}}-2 b \sigma(1+r)^{\sigma-1} \varphi(|\nabla u|)+\right. \\
\left.\quad+\frac{K b}{q}(1+r)^{\sigma-\mu}\right\} .
\end{gathered}
$$

We use again the Claim and (2.34) to deduce that, for $0<\tau<1,0<\Lambda \leq$ $\frac{1-\tau}{2 A^{1 / \delta}}$ and

$$
q=\frac{\tau^{\delta} K b(1+\delta)^{1+\delta}}{A \delta^{\delta}(2 b \sigma)^{1+\delta}}
$$

on the set $\Omega_{\gamma}$ the inequality

$$
A^{-\frac{1}{\delta}} \varphi(|\nabla u|)^{1+\frac{1}{\delta}}-2 b \sigma(1+r)^{\sigma-1} \varphi(|\nabla u|)+\frac{K b}{q}(1+r)^{\sigma-\mu} \geq \Lambda \varphi(|\nabla u|)^{1+\frac{1}{\delta}}
$$

is true, whence

$$
\begin{aligned}
\operatorname{div} W \geq & -(1+\delta) \psi^{\delta} \lambda(u) F(v, r) \varphi(|\nabla u|)|\nabla \psi| \\
& +\Lambda \psi^{1+\delta} \lambda(u)\left|F^{\prime}(v)\right| \varphi(|\nabla u|)^{1+\frac{1}{\delta}} .
\end{aligned}
$$

Having fixed $d>d_{0}$, according to (2.32) there exists a sequence $\left\{R_{k}\right\} \uparrow+\infty$ with $\vartheta R_{k+1}>R_{k}$ such that

$$
\operatorname{vol}\left(B_{R_{k}}\right) \leq c R_{k}^{d}
$$

for some $c>0$. Proceeding as in Theorem 2.1 we arrive to

$$
0<\liminf _{k \rightarrow+\infty} b^{-q}\left(1+\vartheta R_{k}\right)^{-q \sigma}\left(1+R_{k}\right)^{\delta \sigma} R_{k}^{d-(1+\delta)}
$$

and this forces

$$
\delta(\sigma-1)+d-1 \geq q \sigma
$$


Hence we get a contradiction, and thus $K \leq 0$, if $\delta(\sigma-1)+d_{0}-1<0$. In case $\delta(\sigma-1)+d_{0}-1 \geq 0,(2.36)$ and (2.35) lead to

$$
A \delta^{\delta}(2 b \sigma)^{1+\delta}[\delta(\sigma-1)+d-1] \geq \tau^{\delta} K b(1+\delta)^{1+\delta} \sigma
$$

and when $\tau \uparrow 1^{-}, b \downarrow[\max (\hat{u} ; 0)]$ we finish the proof of the case $\sigma>0$.

When $\sigma=0$ we follow the notations and the steps in the proof of Theorem 2.2, working with the vector field $W=\psi^{1+\delta} \lambda(u) F(v, r) T u$ obtained with the choice

$$
F(v, r)=(1+r)^{-q} v^{-q}, \quad q>0 .
$$

The conclusion

$$
\inf _{\Omega_{\gamma}}[1+r(x)]^{\mu} \operatorname{div}(T u)(x) \leq 0
$$

is then a consequence of this choice and of the reasoning cited above.

Again, Theorem 1.2 is a special case of Theorem 2.3, with $\varphi(t)=t$, and $A=\delta=1$.

The argument in the proofs of Theorems 2.1, 2.2, 2.3 is an improvement of original ideas in [14] and [15].

Corollary 2.4 Let $(M,\langle\cdot, \cdot\rangle)$ be a complete manifold with

$$
\liminf _{r \rightarrow+\infty} \frac{\log \operatorname{vol}\left(B_{r}\right)}{r^{1+\delta-\delta \sigma-\mu}}<+\infty
$$

where $\mu \in \mathbb{R}, \sigma \geq 0$ and

$$
\delta \sigma+\mu<1+\delta
$$

Suppose that, for $b(x) \in \mathcal{C}^{0}(M)$,

$$
\text { i) } b(x)>0 \quad \text { on } M ; \quad \text { ii) } b(x) \geq \frac{B}{r(x)^{\mu}} \quad \text { on } M \backslash B_{R_{0}}
$$

for some $R_{0}, B>0$. Let $f \in \mathcal{C}^{0}(\mathbb{R})$ with

$$
\liminf _{t \rightarrow+\infty} f(t)>0 \text {. }
$$

Let $u \in \mathcal{C}^{2}(M)$ be such that $|\nabla u|^{-1} \varphi(|\nabla u|) \nabla u$ is a vector field at least of class $\mathcal{C}^{1}$, and $u$ be a solution of

$$
\operatorname{div}\left(|\nabla u|^{-1} \varphi(|\nabla u|) \nabla u\right) \geq b(x) f(u) \quad \text { on } M
$$

satisfying

$$
u(x)=o\left(r(x)^{\sigma}\right) \quad \text { as } r(x) \rightarrow+\infty .
$$

Then $u^{*}=\sup _{M} u<+\infty$ and $f\left(u^{*}\right) \leq 0$. 
Proof: Without loss of generality we can suppose, using (2.39), that $b(x)$ satisfies

$$
b(x) \geq \frac{\tilde{B}}{[1+r(x)]^{\mu}} \quad \text { on } M
$$

for some appropriate constant $\tilde{B}>0$. First we show that $u^{*}<+\infty$. Because of (2.40) there exist $T>0, c>0$ such that

$$
f(t) \geq c, \quad t \geq T
$$

If $u^{*}=+\infty$,

$$
\Omega_{T}=\{x \in M: u(x)>T\} \neq \emptyset
$$

and, using (2.41), (2.43) and (2.44)

$$
\operatorname{div}\left(|\nabla u|^{-1} \varphi(|\nabla u|) \nabla u\right) \geq \frac{c \tilde{B}}{[1+r(x)]^{\mu}} \text { on } \Omega_{T} .
$$

However, conditions (2.37), (2.38), (2.42) allow to apply Theorem 2.1 with $\hat{u}=0$. Hence, by $(2.5)$,

$$
\inf [1+r]^{\mu} \operatorname{div}(T u) \leq 0
$$

contradicting (2.45).

Having shown that $u^{*}<+\infty$, we can apply Theorem 2.2 to deduce the existence of a sequence $\left\{x_{k}\right\} \subset M$ with the following properties

$$
u\left(x_{k}\right)>u^{*}-\frac{1}{k} \quad ; \quad\left[1+r\left(x_{k}\right)\right]^{\mu} \operatorname{div}(T u)\left(x_{k}\right)<\frac{1}{k} .
$$

Thus

$$
\begin{aligned}
\frac{\tilde{B}}{\left[1+r\left(x_{k}\right)\right]^{\mu}} f\left(u\left(x_{k}\right)\right) & \leq b\left(x_{k}\right) f\left(u\left(x_{k}\right)\right) \\
& \leq \operatorname{div}(T u)\left(x_{k}\right)<\frac{1}{k} \frac{1}{\left[1+r\left(x_{k}\right)\right]^{\mu}}
\end{aligned}
$$

that is

$$
\tilde{B} f\left(u\left(x_{k}\right)\right)<\frac{1}{k} .
$$

Continuity of $f$ and (2.46) then imply $f\left(u^{*}\right) \leq 0$.

Corollary 1.3 of the Introduction is a special case of Corollary 2.4. 


\section{Sharpness of the results and further comments}

We recall that Theorems 2.1 and 2.2 state that, under suitable conditions, one has

$$
\inf _{\Omega_{\gamma}}[1+r(x)]^{\mu} \operatorname{div}(T u)(x) \leq A d_{0}[\max (\hat{u} ; 0)]^{\delta} C(\sigma, \mu, \delta)
$$

where $C \equiv C(\sigma, \mu, \delta)$ is equal to

$$
C= \begin{cases}\left(\frac{2}{1+\delta}\right)^{1+\delta} \delta^{\delta}(1+\delta-\delta \sigma-\mu) \sigma^{\delta} & \text { if } \sigma>0,(1+\delta)(1-\sigma) \leq \mu \\ \left(\frac{2}{1+\delta}\right)^{1+\delta} \delta^{\delta}(1+\delta-\delta \sigma-\mu)^{1+\delta} & \text { if } \sigma>0,(1+\delta)(1-\sigma)>\mu \\ 0 & \text { if } \sigma=0\end{cases}
$$

We note that if $\sigma>0$ and $(1+\delta)(1-\sigma)>\mu$ the value of $C(\sigma, \mu, \delta)$ in (3.1) ii) is larger than that in (3.1)i). These different values amount to different choices of the test functions $F(v, r)$ in the proof of Theorem 2.1. The following example will show that, for the Laplace-Beltrami operator, the correct value of the constant $C$ should be, for $\sigma>0$,

$$
C(\sigma, \mu, 1)=(2-\sigma-\mu) \sigma
$$

that is $(3.1) i)$. In case $\sigma=0$, the constant is clearly sharp.

We construct a model in the sense of Greene and $\mathrm{Wu}[G W]$. Let $M=$ $[0,+\infty) \times S^{1}$. In polar coordinates $(r, \vartheta)$, we define on $(0,+\infty) \times S^{1}$

$$
\langle\cdot, \cdot\rangle=d r^{2}+g(r)^{2} d \vartheta^{2}
$$

where $d \vartheta^{2}$ is the canonical metric on $S^{1}$ and $g(r) \in \mathcal{C}^{\infty}([0,+\infty))$, is positive on $(0,+\infty)$ and it satisfies

$$
g(r)= \begin{cases}r & \text { on }[0,1] \\ r^{1-\sigma-\mu} \exp \left(d_{0} r^{2-\sigma-\mu}\right) & \text { on }[2,+\infty)\end{cases}
$$

for some parameters $\sigma \geq 0, \mu \in \mathbb{R}, d_{0}>0$ such that

$$
2-\sigma-\mu>0 \text {. }
$$

We note that, because of the behaviour of $g$ near the origin, the metric $\langle\cdot, \cdot\rangle$ in (3.2) extends to a smooth, obviously complete, metric on all of $M=\mathbb{R}^{2}$ that we shall again denote with $\langle\cdot, \cdot\rangle$. Computations, using (3.3), show

$$
\lim _{r \rightarrow+\infty} \frac{\log \operatorname{vol}\left(B_{r}\right)}{r^{2-\sigma-\mu}}=d_{0} .
$$


For $\rho \in \mathbb{R}$ we define

$$
u(x)=\int_{0}^{r(x)} g(t)^{-1}\left\{\int_{0}^{t}(1+s)^{-\rho} g(s) d s\right\} d t .
$$

Then $u$ is a $\mathcal{C}^{2}(M)$, non-negative, radial function such that

$$
\Delta u(x)=[1+r(x)]^{-\rho} \text { on } M .
$$

Computations, again using (3.3), show that, if $\mu+\sigma-\rho \neq 0$,

$$
\frac{u(r)}{r^{\sigma}} \sim \frac{r^{\mu-\rho}}{\sigma d_{0}(2-\sigma-\mu)} \quad \text { as } r \rightarrow+\infty .
$$

Therefore, if $\rho=\mu$, using the notations of Theorem 2.1, we have

$$
\hat{u}=\frac{1}{\sigma d_{0}(2-\sigma-\mu)}
$$

and

$$
[1+r(x)]^{\mu} \Delta u(x) \equiv 1 .
$$

This shows that the bound in (1.16) of Theorem 1.1 is sharp if $2(1-\sigma) \leq \mu$.

$$
\text { If } \mu-\rho>0
$$

$$
\limsup _{r \rightarrow+\infty} \frac{u(x)}{r(x)^{\sigma}}=+\infty
$$

while (2.3) is still valid. However, according to (3.4), having fixed $N>0$, choosing $\gamma$ sufficiently large yields $\Omega_{\gamma} \neq \emptyset$ and

$$
\inf _{\Omega_{\gamma}}[1+r]^{\mu} \Delta u \geq N .
$$

This shows that the exponent $\sigma$ in (2.4) cannot be taken smaller. The same reasoning shows that the exponent in the volume growth (2.3) cannot be taken smaller.

We recall that Theorem 2.3 states that

$$
\inf _{\Omega_{\gamma}}[1+r(x)]^{\mu} \operatorname{div}(T u)(x) \leq A[\max (\hat{u} ; 0)]^{\delta} \sigma^{\delta} C\left(\sigma, \delta, d_{0}\right)
$$

where $C=C\left(\sigma, \delta, d_{0}\right)$ is equal to

$$
C= \begin{cases}\left(\frac{2}{1+\delta}\right)^{1+\delta} \delta^{\delta}\left[\delta(\sigma-1)+d_{0}-1\right] & \text { if } \delta(\sigma-1)+d_{0}-1 \geq 0 \\ 0 & \text { if } \delta(\sigma-1)+d_{0}-1<0\end{cases}
$$


An analysis similar to the above can be developped in this case too. Let us only point out that, for the Laplacian, estimate (3.5) $i$ ) is sharp. Indeed, in $\mathbb{R}^{2}$ with its canonical metric, the function $u(x)=r(x)^{2}$ satisfies $\Delta u(x) \equiv 4$ (here $A=\delta=1, \sigma=2, \mu=0$ ).

Similarly, $u(x)=r(x)^{2+\varepsilon}$ for some $\varepsilon>0$ shows that the exponent $\sigma=2$ in $(2.33)$ is best possible.

Next we show that assumption (2.38) of Corollary 2.4 can not be weakened. For instance, we consider $\mathbb{H}^{m}$, the hyperbolic $m$-dimensional space with its canonical metric of constant sectional curvature -1 , that we realize as the model $[0,+\infty) \times S^{m-1}$ with metric, in polar coordinates $(r, \vartheta)$, $\langle\cdot, \cdot\rangle=d r^{2}+(\sinh r)^{2} d \vartheta^{2}$. Note that

$$
\lim _{r \rightarrow+\infty} \frac{\log \operatorname{vol}\left(B_{r}\right)}{r}=m-1 .
$$

For $a \in(0, m-1]$ let $u \in \mathcal{C}^{2}\left(\mathbb{H}^{m}\right)$ be the radial function defined by

$$
u(x)=\int_{0}^{r(x)} \frac{a(\sinh t)^{1-m} \int_{0}^{t}(\sinh s)^{m-1} d s}{\left\{1-(\sinh t)^{2(1-m)}\left[a \int_{0}^{t}(\sinh s)^{m-1} d s\right]^{2}\right\}^{1 / 2}} d t .
$$

A simple verification shows that $u$ satisfies

$$
\operatorname{div}\left(\frac{\nabla u}{\sqrt{1+|\nabla u|^{2}}}\right)=a \text { on } \mathbb{H}^{m} .
$$

Furthermore

$$
\frac{u(x)}{r(x)} \rightarrow 1 \quad \text { as } r \rightarrow+\infty .
$$

In this case $\varphi(t)=t\left(1+t^{2}\right)^{-1 / 2} \leq t$ on $[0,+\infty)$, so that in the structural conditions (2.1) iii) we can take $A=\delta=1$. Choosing $b(x) \equiv a$ on $M$, $f(t) \equiv 1$, we see that all the assumptions of Corollary 2.4 are satisfied, but (2.42) barely fails to be met.

Substituting $\mathbb{H}^{m}$ with a manifold $(M,\langle\cdot, \cdot\rangle)$ we can geometrically interpret equation (3.6) as the condition for the graph of

$$
\Gamma_{u}: M \rightarrow M \times \mathbb{R}
$$

given by $\Gamma_{u}(x)=(x, u(x))$ to have constant mean curvature $a / m$. It is an old problem to determine the class of non-admissible functions $b(x)$ for the prescribed mean curvature equation

$$
\operatorname{div}\left(\frac{\nabla u}{\sqrt{1+|\nabla u|^{2}}}\right)(x)=b(x) \quad \text { on } M .
$$


The above example shows that some restriction on the growth of $u$ need to be imposed.

Applying Corollary 2.4 we have

Proposition 3.1 Let $(M,\langle\cdot, \cdot\rangle)$ be a complete manifold with

$$
\liminf _{r \rightarrow+\infty} \frac{\log \operatorname{vol}\left(B_{r}\right)}{r^{2-\sigma-\mu}}<+\infty
$$

where $\mu \in \mathbb{R}, \sigma \geq 0$ and $\sigma+\mu<2$. Suppose that, for $b(x) \in \mathcal{C}^{0}(M)$,

$$
\text { i) } b(x)>0 \quad \text { on } M ; \quad \text { ii) } b(x) \geq \frac{B}{r(x)^{\mu}} \quad \text { on } M \backslash B_{R_{0}}
$$

for some $R_{0}, B>0$. Then, for any $\varepsilon>0$ there are no graphs of mean curvature $b(x) / m$ lying between the graphs of

$$
-\Gamma_{\sigma-\varepsilon}: x \mapsto\left(x,-(r(x))^{\sigma-\varepsilon}\right) \quad \text { and } \quad \Gamma_{\sigma-\varepsilon}: x \mapsto\left(x,(r(x))^{\sigma-\varepsilon}\right) \text {. }
$$

\section{References}

[1] Coulhon, T., Holopainen, I. and Saloff-Coste, L.: Harnack inequality and hyperbolicity for subelliptic $p$-Laplacians with applications to Picard type theorems. Geom. Funct. Anal. 11 (2001), no. 6, 1139-1191.

[2] Cheng, S. Y. and Yau, S. T.: Differential equations on Riemannian manifolds and their geometric applications. Comm. Pure Appl. Math. 28 (1975), no. 3, 333-354.

[3] Grigor'yan, A.: Analytic and geometric background of recurrence and non-explosion of the Brownian motion on Riemannian manifolds. Bull. Amer. Math. Soc. (N.S.) 36 (1999), no. 2, 135-249.

[4] Grigor'yan, A. AND YaU, S. T.: Isoperimetric properties of higher eigenvalues of elliptic operators. Amer. J. Math. 125 (2003), no. 4, 893-940.

[5] Greene, R. E. And Wu, H.: Function theory on manifolds which possess a pole. Lecture Notes in Mathematics 699. Springer, Berlin, 1979.

[6] Holopainen, I.: A sharp $L^{q}$-Liouville theorem for $p$-harmonic functions. Israel J. Math. 115 (2000), 363-379.

[7] Holopainen, I.: Volume growth, Green's function, and parabolicity of ends. Duke Math. J. 97 (1999), no. 2, 319-346.

[8] KARP, L.: Differential inequalities on complete Riemannian manifolds and applications. Math. Ann. 272 (1985), no. 4, 449-459.

[9] Pigola, S., Rigoli, M. and Setti, A. G.: A remark on the maximum principle and stochastic completeness. Proc. Amer. Math. Soc. 131 (2003), no. $4,1283-1288$. 
[10] Pigola, S., Rigoli, M. and Setti, A. G.: Volume growth, "a priori" estimates and geometric applications. Geom. Funct. Anal. 13 (2003), no. 6, 1302-1328.

[11] Pucci, P. And Serrin, J.: A note on the strong maximum principle for elliptic differential inequalities. J. Math. Pures Appl. 79 (2000), 57-71.

[12] Pucci, P., Serrin, J. And Zou, H.: A strong maximum principle and a compact support principle for singular elliptic inequalities. J. Math. Pures Appl. 78 (1999), 769-789.

[13] Ratto, A., Rigoli, M. and Setti, A. G.: On the Omori-Yau maximum principle and its applications to differential equations and geometry. J. Funct. Anal. 134 (1995), no. 2, 486-510.

[14] Rigoli, M. and Setti, A. G.: Liouville type theorems for $\varphi$-subharmonic functions. Rev. Mat. Iberoamericana 17 (2001), no. 3, 471-520.

[15] Rigoli, M., Salvatori, M. And Vignati, M.: A Liouville type theorem for a general class of differential operators on complete manifolds. Pacific J. Math. 194 (2000), no. 2, 439-453.

[16] Serrin, J.: Entire solutions to nonlinear Poisson equations. Proc. London Math. Soc. (3) 24 (1972), 348-366.

[17] Sturm, K. T.: Analysis on local Dirichlet spaces I. Recurrence, conservativeness and $L^{p}$-Liouville properties. J. Reine Angew. Math. 456 (1994), 173-196.

[18] YAu, S. T.: A general Schwarz lemma for Kähler manifolds. Amer. J. Math. 100 (1978), no. 1, 197-203.

Recibido: 21 de enero de 2003.

Marco Rigoli

Dipartimento di Matematica

Università di Milano

Via Saldini 50, 20133-Milano, Italy

marco.rigoli@mat.unimi.it

Maura Salvatori

Dipartimento di Matematica

Università di Milano

Via Saldini 50, 20133-Milano, Italy

maura.salvatori@mat .unimi.it

Marco Vignati

Dipartimento di Matematica

Università di Milano

Via Saldini 50, 20133-Milano, Italy

marco.vignati@mat.unimi.it 